\title{
RESEARCH HIGHLIGHT CAR T cells: from tinkering to rational design
}

\author{
Bernard Malissen (iD) ${ }^{1,2}$ \\ Cell Research (2020) 30:948-949; https://doi.org/10.1038/s41422-020-00420-6
}

\begin{abstract}
Hartl et al. and Wu et al. revealed that the CD3\& subunit of the TCR-CD3 complex - a central player of T cell activation - contains previously unrecognized signaling motifs that permit its functional coupling to tyrosine protein kinases. When grafted into chimeric antigen receptors (CARs), such signaling motifs enhance the antitumor activity of CAR T cells, a promising class of anti-tumoral therapeutics.
\end{abstract}

$\mathrm{T}$ cells are central participants in adaptive immunity. They express $T$ cell antigen receptors (TCRs) on their surface, through which they detect antigens in a highly sensitive manner. When the TCR binds to an antigen, the immunoreceptor tyrosine-based activation motifs (ITAMs) found in the TCR-associated CD3 chains are phosphorylated by the protein tyrosine kinase (PTK) Lck. This allows the recruitment and activation of the cytosolic PTK ZAP70 that phosphorylates the transmembrane adapter LAT, leading to the assembly of a multiprotein complex that ensures propagation and diversification of TCR signals. ${ }^{1}$

The ITAMs found in the $\operatorname{CD} 3 \delta, \gamma, \varepsilon$ and $\zeta$ chains originate from a common ancestral building block and once bi-phosphorylated, bind with high affinity to the tandem $\mathrm{SH} 2$ domains found in ZAP70. ${ }^{2}$ In the process of evolution, some CD3 chains have acquired some additional functions. For instance, CD3y comprises a di-leucine-based internalization motif, whereas $C D 3 \varepsilon$ possesses a proline-rich sequence (PRS) required for the recruitment of the adapter protein NCK. In addition, basic residue-rich sequences (BRS) in $\mathrm{CD} 3 \varepsilon$ and $\mathrm{CD} 3 \zeta$ bind to acidic phospholipids in the membrane to regulate TCR signaling.

$T$ cells expressing chimeric antigen receptors (CARs) demonstrate remarkable efficacy against certain B-cell malignancies. CARs consist of extracellular domains made of single-chain $\mathrm{V}$ fragments (scFv) specific for cancer-associated cell surface antigens connected via diverse hinge-transmembrane segments to cytoplasmic segments comprising CD3 ITAMs combined with various intracellular domains of costimulatory molecules (CD28, CD27, CD134, CD137, or ICOS). On an individual basis, CARs are 10-1000-fold less sensitive than TCRs, and as a result CAR T cells fail to exert antitumor activity in conditions of low target antigen density resulting in tumor immune escape., ${ }^{3,4}$ Attempting to increase CAR sensitivity might, however, coincidently enhance CAR T cell toxicity and impede their long-term persistence via both tonic signaling during ex vivo expansion and exhaustion in response to in vivo chronic antigen stimulation.

Two recent studies show how a better understanding of TCR biology might help designing better CARs. In a first study, Hartl

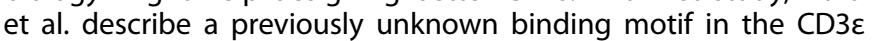
cytoplasmic tail that is denoted as the receptor kinase (RK) motif and conserved in vertebrates. ${ }^{5}$ Upon interacting in a noncanonical mode with the Lck SH3 domain, the RK motif augments local Lck activity, optimal ITAM phosphorylation and T cell activation. CD3E sequences encompassing the RK motif or a mutant RK motif with reduced Lck binding were inserted between the 4-1BB and CD3Z sequences of an anti-CD19 CAR. Both motifs enhanced in vivo tumor control by CAR T cells in a preclinical mouse model. On that basis, Hartl et al. suggest that the RK motif boosts both TCR and CAR signaling due to direct recruitment of Lck and irrespective of Lck association with coreceptors. In a second study, Wu et al. ${ }^{6}$ used mass spectrometry to simultaneously quantitate ITAM phosphorylation of all CD3 chains upon TCR stimulation. A subpopulation of CD3E ITAMs was found to be mono-phosphorylated, and capable of recruiting the CSK PTK to attenuate Lck activity and in turn TCR signaling. This differs from previous models in which CSK was thought to be brought in contiguity to Lck via binding to the PAG transmembrane adapter. In addition, they showed that the $\mathrm{SH} 3$ domain of the $\mathrm{p} 85$ regulatory subunit of class IA phosphatidylinositol 3-kinase (PI3K) interacted with the CD3 $\varepsilon$ BRS motif to enhance PI3K/AKT signaling following TCR activation. Wu et al. next tested the in vivo antitumor activity of a second-generation CAR carrying the $\mathrm{CD} 3 \varepsilon$ cytosolic domain using a mouse model of $B$ cell lymphoma and showed that they exerted a better tumor control as compared to those deprived of $\mathrm{CD} 3 \varepsilon$ cytosolic domain. Mechanistically, the CSK-recruiting monophosphorylated ITAM of CD3E was proposed to reduce CAR-T cytokine production whereas the BRS of CD3E promoted CAR-T persistence via 885 recruitment. Therefore, according to $\mathrm{Wu}$ et al.

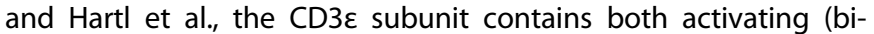
phosphorylated ITAM, BRS and RK) and inhibitory (monophosphorylated ITAM) motifs.

Current lines of research in CAR T cell therapeutics also concur to address other major barriers to progress. Activated $\mathrm{T}$ cell populations responding to tumors can differentiate into discrete subsets of short-lived effector T cells, long-lived memory $T$ cells, and exhausted T cells. Accordingly, attempts to engineer exhaustion-resistant CAR T cells and to balance memory versus effector cell fate decision have been also addressed. ${ }^{7}$ However, these important advances might still be plagued by the observation that the CAR architecture originally designed by Zelig Eshhar affords much flexibility in clinical applications but fails to reproduce the dynamics of TCR-CD3 signaling. For instance, a recent study of CAR $T$ cell activation using single-molecule microscopy revealed that CARs poorly convert ligand binding into early signaling events, such as ITAM phosphorylation, recruitment and activation of ZAP70 kinase and subsequent release of ZAP70 to amplify and disperse signals. ${ }^{4}$ Even when CD8 binding had been abrogated, antigen recognition via TCRs still proved to be 50 times more sensitive than that mediated by CARs. In view of the remarkable ability of $T$ cells to respond to even a single antigen molecule, an interesting alternative to CARs has been the development of TCR constructs comprising a

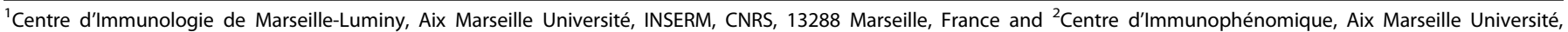
INSERM, CNRS, 13288 Marseille, France

Correspondence: Bernard Malissen (bernardm@ciml.univ-mrs.fr)

Published online: 14 October 2020 
tumor-specific scFv fused at the N-terminus of one TCR chain. ${ }^{8}$ However, even in that design, the use of scFv with a long half-life for its cognate antigen should hamper serial triggering and be detrimental to $\mathrm{T}$ cell activation in condition of low target antigen density. ${ }^{9}$

Despite the enormous amount of information concerning the nature of molecules involved in the TCR signal transduction network, ${ }^{10}$ the description at the nanoscale levels of the events leading to productive TCR engagements, ${ }^{11}$ and the cryo-electron microscopy structure of a TCR-CD3 complex containing the extracellular domains and all the transmembrane helices, ${ }^{12}$ the mechanisms by which information is passed from the TCR antigen-binding site through the plane of the membrane and results in the earliest events of $\mathrm{T}$ cell activation remain highly controversial. Moreover, the importance of distinguishing protein-protein interactions that occur at physiological levels from those that are possible experimentally in conditions of overexpression or of disrupted cellular architecture still remains a major issue in many studies. Therefore, given the considerable challenges that exist in unraveling the mechanisms of TCR triggering and translating them into the design of better CAR
T cells, humility is a more appropriate reaction than hubris. However, as illustrated by the meticulous work of Hartl et al. and $\mathrm{Wu}$ et. al, the prospects of integrating multidisciplinary approaches, each with unique advantages and drawbacks, in unveiling how $T$ cells carry out the formidable task of identifying small numbers of foreign antigenic peptides and in translating it to CAR T cell design are excellent.

\section{REFERENCES}

1. Shah, N. H. et al. Elife 5, e20105 (2016).

2. Wegener, A. M. et al. Cell 68, 83-95 (1992).

3. Harris, D. T. et al. J. Immunol. 200, 1088-1100 (2018).

4. Gudipati, V. et al. Nat. Immunol. 21, 848-856 (2020).

5. Hartl, F. A. et al. Nat. Immunol. 21, 902-913 (2020).

6. Wu, W. et al. Cell 182, 855-871 (2020).

7. Lynn, R. C. et al. Nature 576, 293-300 (2019).

8. Baeuerle, P. A. et al. Nat. Commun. 10, 2087 (2019).

9. Malissen, B. Nat. Immunol. 2, 196-198 (2001).

10. Voisinne, G. et al. Nat. Immunol. 20, 1530-1541 (2019).

11. Lin, J. J. Y. et al. Sci. Signal. 12, eaat8715 (2019).

12. Dong, D. et al. Nature 573, 546-552 (2019). 